\title{
CAUSATION AT DIFFERENT LEVELS: Tracking the commitments of mechanistic explanations
}

\author{
Peter Fazekas \\ School of Philosophy, Psychology and Language Sciences, \\ The University of Edinburgh; \\ Institute for Philosophical Research, Hungarian Academy of Sciences \\ email: P.Fazekas@sms.ed.ac.uk
}

\section{Gergely Kertész}

Department of Philosophy and History of Science, Budapest University of Technology and Economics

email: rumcais@gmail.com

- penultimate draft —

forthcoming in Biology and Philosophy

\begin{abstract}
This paper tracks the commitments of mechanistic explanations focusing on the relation between activities at different levels. It is pointed out that the mechanistic approach is inherently committed to identifying causal connections at higher levels with causal connections at lower levels. For the mechanistic approach to succeed a mechanism as a whole must do the very same thing what its parts organised in a particular way do. The mechanistic approach must also utilise bridge principles connecting different causal terms of different theoretical vocabularies in order to make the identities of causal connections transparent.

These general commitments get confronted with two claims made by certain proponents of the mechanistic approach: William Bechtel often argues that within the mechanistic framework it is possible to balance between reducing higher levels and maintaining their autonomy at the same time, whereas, in a recent paper, Craver and Bechtel argue that the mechanistic approach is able to make downward causation intelligible.

The paper concludes that the mechanistic approach imbued with identity statements is no better candidate for anchoring higher levels to lower ones while maintaining their autonomy at the same time than standard reductive accounts are, and that what mechanistic explanations are able to do at best is showing that downward causation does not exist.
\end{abstract}

Keywords: Mechanistic explanation, reduction, autonomy, downward causation 


\section{CAUSATION AT DIFFERENT LEVELS: Tracking the commitments of mechanistic explanations}

\section{Introduction}

We are in an era of mechanisms (again). There are enthusiastic people around us saying that mechanisms are everywhere. There is a mechanism explaining how keys open locks (Glennan 2009), how the DNA gets replicated, or how chemical transmission happens at synapses (Machamer \& Darden \& Craver 2000). There are deep interconnected levels of mechanisms explaining the whole brain itself (Craver 2007, p. 7). In fact, some argue, causation per se (at non-fundamental levels) ought to be understood as a mechanism producing the appropriate effect (Glennan 1996).

This new mechanistic approach makes at least two points. The first point is a methodological one: it is argued that what scientists (typically life scientists) do is exploring and identifying mechanisms. The second point is an explanatory one: it is claimed that understanding something properly (and thus explaining a phenomenon adequately) amounts to comprehending the mechanism responsible for the phenomenon.

But sometimes a third point is also made. William Bechtel, one of the leading advocates of the mechanistic approach, argues that mechanistic explanations are able to reconcile two quite antagonistic claims about the status of special sciences: their reducibility and independence (Bechtel 2006, 2007, 2008). Contrary to the received view, according to which reductive ambitions are usually seen as threatening the independence of special sciences, Bechtel argues that these two ways of looking at the special sciences_-once reformulated within the mechanistic framework-are in fact compatible.

In what follows, first, we shall focus on what mechanistic explanations say about causation at different levels. In particular, we shall analyse their explicit and implicit commitments concerning the relationship between what a mechanism as a whole does and what the constituents as parts of an organised structure do. By showing that it is one of the fundamental tenets of the mechanistic approach that causal roles of higher levels are identical with certain causal roles of lower levels we conclude that, contrary to how it is often put in the literature, the mechanistic approach deploys identity statements about causal roles and even relies on bridge laws. 
Second, we shall argue that these commitments ruin William Bechtel's programme. The mechanistic framework is no better candidate for reducing higher levels (or special sciences) and maintaining their autonomy at the same time than any other reductive approach. We show that Bechtel's reasons supporting the independence of higher levels are incompatible with the commitments of the mechanistic endeavour.

Finally, we shall confront these commitments with a recent claim made by Carl Craver and William Bechtel (Craver \& Bechtel 2007). They argue that within the mechanistic framework it is possible to render the mysterious notion of downward causation intelligible via identifying it with so-called mechanistically mediated effects. We demonstrate that, due to its commitments to identity statements, what the mechanistic approach is able to achieve, at best, is explaining downward causation away-i.e. showing that the phenomenon as characterised by e.g. emergentists does not exist.

That is, we make and defend three claims. The first claim is a general one, which applies across the board to all versions of mechanistic explanations. The second and third claims, on the other hand, are particular critical notes targeting special applications of the mechanistic framework, and thus do not necessarily generalise over to other proponents of the approach.

\section{The Mechanistic Approach}

In this section we set the stage for our analysis. First, a general characterisation of the mechanistic approach is introduced, followed by a brief summary of the motivation behind and the main arguments supporting the claims proposed by Bechtel (2006, 2007, 2008) and Craver and Bechtel (2007).

\subsection{General characterisation}

The mechanistic approach identifies a phenomenon via identifying the tasks performed-i.e. the causal roles played - by a system producing the phenomenon. The very point of the mechanistic approach is to explain a phenomenon by understanding the mechanism responsible for these tasks.

Understanding the relevant mechanism consists in decomposing the system into parts, specifying the properties and causal roles of the parts, and understanding the spatial and temporal organisation of the parts. The mechanistic approach employs two interdependent building blocks for characterising mechanisms: entities and activities. Entities are the parts composing the mechanism, their 
activities are in virtue of what they contribute to the working of the mechanism. A mere aggregate of entities and their activities is not a mechanism, however. Entities and activities must be arranged into a specific spatial and temporal order otherwise they would not be able to perform a certain task together. That is, the mechanistic approach tries to account for the phenomenon (the explanandum) in terms of the organised activity of the parts constituting the system.

Consider Figure $1^{1}$. S $\psi$-ing is the phenomenon in question. The arrows indicate how $\mathrm{S} \psi$ ing is connected to its context, i.e. to other entities at its level. According to the mechanistic approach, $\mathrm{S} \psi$-ing can be accounted for in terms of the components $\mathrm{X}_{1}, \mathrm{X}_{2}, \mathrm{X}_{3}, \mathrm{X}_{4}$ and $\mathrm{X}_{5}$, and their activities ( $\Phi_{1}$-ing, $\Phi_{2}$-ing, $\Phi_{3}$-ing, $\Phi_{4}$-ing, and $\Phi_{5}$-ing respectively) organised in an appropriate way. These entities and activities organised in the appropriate way constitute the mechanism responsible for the $\psi$-ing of S.

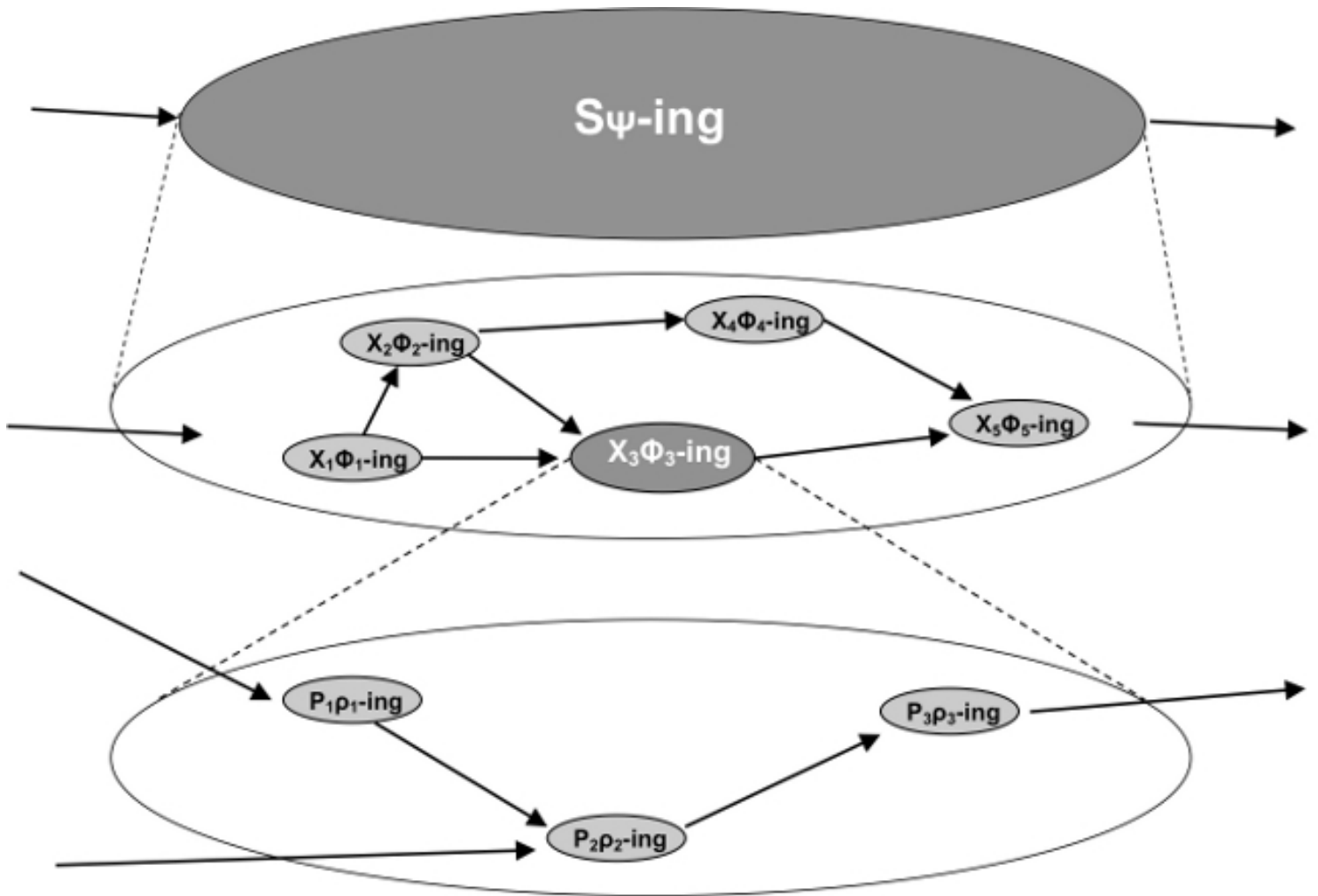

Fig. 1 The general scheme of mechanistic explanations.

S performs the task $\psi$-ing. On descending levels it is decomposed into the organised activities of its constituent parts.

\footnotetext{
${ }^{1}$ Figure 1 is based on Figure 2 in (Craver 2001, p. 66) and Figure 1.1 in (Craver 2007, p. 7).
} 
As it is shown on Figure 1, the mechanistic approach is a multilevel approach. Once the $\psi$ ing of $\mathrm{S}$ is decomposed into the lower level organised structure of entities $\left(\mathrm{X}_{\mathrm{i}}-\mathrm{S}\right)$ and their activities ( $\Phi_{\mathrm{i}-\mathrm{ing}}$ ) it is possible to apply the same methodology again in order to account for the $\Phi_{\mathrm{i}}$-ing of $\mathrm{X}_{\mathrm{i}}$-S in terms of a still lower level mechanism. So, for example, the $\Phi_{3}$-ing of $X_{3}$ can be accounted for in terms of the organised $\rho_{\mathrm{i}}$-ing of some $\mathrm{P}_{\mathrm{i}} . \mathrm{P}_{1} \rho_{1}$-ing, $\mathrm{P}_{2} \rho_{2}$-ing, and $\mathrm{P}_{3} \rho_{3}$-ing (organised in an appropriate way) together constitute the mechanism responsible for the $\Phi_{3}$-ing of $\mathrm{X}_{3}$.

\subsection{Bechtel's way of defending autonomy}

As we have seen, mechanistic explanations account for higher level phenomena in terms of lower level entities and activities. Questions concerning the relationship between these levels-especially if explanations at different levels utilise different theoretical vocabularies, i.e. belong to different special sciences (cf. section 3.2) — thus naturally arise.

In contemporary literature there is a number of different approaches to this problem. At one end of the spectrum, there is, for example, John Bickle, whose 'ruthless reductionism' argues that lower level accounts have an explanatory primacy in the sense that it is the lower level account which really explains a higher level phenomenon-once lower level explanations are completed one does not need higher level explanations anymore (Bickle 2003, 2006). At the other end of the spectrum, there is Peter Menzies, who not just denies the explanatory primacy of lower levels, but even claims that higher levels are causally autonomous - it is the higher level entity rather than the lower level one which is, according to him, causally efficient in bringing about a certain effect (Menzies 2003, 2010).

William Bechtel tries to balance between these two extremes. ${ }^{2}$ On the one hand, he argues that the mechanistic approach is reductive since it decomposes higher level wholes into parts and accounts for the task it performs in terms of lower level entities and activities. On the other hand, though, he would also like to maintain the autonomy of higher levels by emphasising that information about the lower level parts alone is not sufficient for understanding why a higher level whole behaves as it does (Bechtel 2007). He argues for this latter claim in the following way:

\footnotetext{
${ }^{2}$ Note that not all proponents of the mechanistic approach can easily be positioned along this continuum. Carl Craver, for example, by arguing for the so-called 'mosaic unity' of the sciences, implies that sciences are neither reduced to one another nor are they autonomous from each other, but rather equally contribute to the development of multilevel mechanisms (Craver 2007).
} 
"The parts of a whole behave in a particular way because of how they are organized in the mechanism. Information about how the parts are organized goes beyond the account of the parts and their operations. Moreover, the mechanism interacts causally with other entities. These interactions provide the input and set the conditions for the mechanism and information about them is not part of the reductive account characterizing the parts and operations within the mechanism. Securing information about both the organisation within the mechanism and the relations between the mechanism and its environment requires going beyond the reductive aspect of mechanistic explanation and incorporating the results of other autonomous inquiries." (Bechtel 2007, pp. 182-183, emphasis added)

Basically, when arguing for the autonomy of higher levels, Bechtel relies on the claim that in order to explain a higher level phenomenon properly, one needs information only present at that higher level. First, Bechtel argues, organisational information, information about how the parts are spatially and temporally organised goes beyond the account of the parts and their operations. Second, contextual information about how the mechanism as a whole is related to other entities in its environment, is also something, which cannot be captured at the level of the parts and their operations.

Consider, for example, identifying the mechanism responsible for the activity of the photoreceptors in the retina (converting light into electrochemical signals). Electromagnetic radiation is absorbed by a photopigment in the membrane of the disks in the outer segment of the photoreceptors. Photopigments are receptor proteins (opsins) with a prebound chemical agonist (retinal, a derivative of vitamin A). The absorption of light causes a change in the conformation of retinal so that it activates the opsin. This stimulates a G-protein in the disk membrane (transducin) which in turn activates an effector enzyme phosphodiesterase (PDE). PDE breaks down cyclic guanosine monophosphate (cGMP), an intracellular second messenger which keeps the sodium channels in the membrane of the photoreceptor open. The reduction in cGMP causes the sodium channels to close and the photoreceptor membrane to hyperpolarize. (Cf. Bear \& Connors \& Paradiso 2001, pp. 283299; Kandel \& Schwartz \& Jessell 2000, pp. 507-515)

What Bechtel argues for is that comprehending e.g. molecular facts about retinal, opsin, transducin, etc. is not sufficient for understanding how the retina works. First, molecular level facts 
about retinal, opsin, etc. carry no information about how these constituents need to be arranged for the retina to be able to convert light into electrochemical signals. Second, molecular level facts also leave out facts about higher level context, i.e. how the retina itself is situated relative to the lens, the optic nerve, etc. These additional information are crucial - it is the specific spatial and temporal organisation of the molecular parts which allow them to trigger each other, and it is the specific way of being embedded in the organisation of the pupil, the lens, the ciliary muscles, etc. which makes it possible for the retina to do its job.

That is, without organisational and contextual information it is impossible to provide a sufficient explanation of a given phenomenon. Since, according to Bechtel, a lower level account in itself falls short in capturing this information, it is insufficient to explain the behaviour of the whole mechanism. Thus-Bechtel concludes - higher levels are autonomous: they capture crucial, and relevant information not captured by any lower level accounts. That is, it is not possible to account for all the facts solely on the basis of the lower level facts.

\subsection{Downward causation through the spectacles of mechanistic explanations}

Generally, downward causation is a phenomenon where a higher level entity exerts causal influence on lower level entities. It is the central notion of emergence (cf. Alexander 1920; Broad 1925; McLaughlin 1992; Morgan 1923), and it is widely discussed both within the philosophical and the scientific literature. However, there is a certain sense of mysteriousness associated to the term. Even the question if downward causation is a coherent notion is hotly debated (Bedau 2002; Kim 1999, 2000; Stephan 2002; Yates 2009). ${ }^{3}$

From our present perspective the problem of downward causation is interesting because if entities at higher levels could exert causal influence on entities at lower levels then that would credit autonomy — in a quite strong sense - to higher levels. In a recent paper, Craver and Bechtel argue that the mechanistic framework is able to make sense of downward causation. Here we are interested in if their account contributes to the autonomy of higher levels.

Craver and Bechtel claim that the correct way of understanding downward causation-and all other versions of inter-level causation - is to interpret them as a hybrid of inter-level constitutive

\footnotetext{
${ }^{3}$ Especially its reflexive variety where a whole exerts causal influence on its parts.
} 
and intra-level causal relations. They call these hybrids mechanistically mediated effects (Craver \& Bechtel 2007).

Their argument proceeds as follows. First Craver and Bechtel claim that the notion of levels, as it occurs in the context of inter-level causation, in fact stands for levels of mechanisms. This makes it legitimate to interpret inter-level causation from the point of view of the mechanistic approach. Next, it is claimed that within the mechanistic framework causation is strictly an intra-level relation. Entities of different levels of mechanisms are in a part-whole relation with each othermechanisms as wholes are constituted by the organised activity of their parts. That is, levels of mechanisms are 'levels of aggregation', 'levels of composition' or 'constitutive levels' (cf. Craver \& Bechtel 2007, p. 550). Constitution is not a causal relation. As Craver and Bechtel put it: "many common assumptions about the nature of causation preclude the possibility of causal relation between parts (components) and wholes (mechanisms)" (Craver \& Bechtel 2007, p. 551).

Thus, literally, there is no such thing as inter-level causation. What there is instead-and according to Craver and Bechtel what is usually misinterpreted as inter-level causation - is the following. First, entities of a given level exert causal influence on other entities of the same level. Second, these effects get mediated down (top-down causation) or up (bottom-up causation) to the next level via the constitution relation. E.g. affecting the behaviour of a component part affects the behaviour of the whole not because the part exerts a direct causal influence on the whole but because the whole is constituted by the organised activity of its parts. Since the activity of every part plays a crucial role in what the mechanism as a whole does changing the activity of a part changes the behaviour of the whole.

Consider the example of the previous section again. It might be tempting to say that the eye transducing the visual stimulus into the language of the nervous system causes the hyperpolarisation of the photoreceptors thus evoking an inter-level causal connection between the eye and the photoreceptor. However, what the mechanistic approach points out is exactly that it is not the case. The eye transducing is a higher level whole which is constituted by the precise organisation of lower level entities (pupil, lens, ciliary muscles, etc.), which themselves are constituted by the organised activity of still lower level entities, (e.g. photoreceptors) etc. The eye transducing the visual stimulus into neural activation patterns does not exert a causal influence on photoreceptors. Photoreceptors are parts of the eye, their activity (hyperpolarisation) contributes to the behaviour of the eye. Craver and Bechtel emphasise that the relation between the behaviour of a whole and its part 
(say, between the eye transducing and a photoreceptor hyperpolarising) - contrary to the relation between entities causally connected-is symmetric: the eye transduces because the photoreceptor hyperpolarises and vice versa (cf. Craver \& Bechtel 2007, p. 554).

\section{What Constitution Amounts to}

The notion of constitution plays a central role within the mechanistic framework - as we have seen, the fundamental claim of the approach is that the organised activity of the parts constitutes the whole. However, understanding what is meant by constitution within the mechanistic framework properly is not straightforward.

Note that, on the one hand, proponents of the mechanistic approach evoke the constitution relation as an alternative to identity (cf. e.g. Craver 2007). To this extent, they are buying in a widely held position. As it is often argued, the lump of clay constitutes the statue, but not vice versa: the statue does not constitute the lump of clay. That is, constitution - contrary to identity-is an asymmetric relation (Baker 1997; Johnston 1992). On the other hand, though, constitution is usually understood as an intra-level relation (cf. e.g. Paul 2007). Compare this with the way the mechanistic approach deploys constitution explicitly as an inter-level relation. The lump of clay and the statue are at the same level of composition/aggregation, whereas a whole and its spatially and temporally organised parts are at different levels of composition/aggregation.

Moreover, consider how Craver and Bechtel claim, on the one hand, that it is constitution that relates a higher level with a lower level, whereas, on the other hand, they claim that the relation between levels is symmetrical. They say:

"The relation is symmetrical precisely because the mechanism as a whole is fully constituted by the organized activities of its parts: a change in the parts is manifest as a change in the mechanism as a whole, and a change in the mechanism is also a change in at least some of its component parts.” (Craver \& Bechtel 2007, p. 554)

Note, however, that the symmetrical relation in question holds between what the mechanism as a whole does and the behaviour of the spatially and temporally organised parts. That is, the symmetrical relation connects the activity of the whole and the organised activity of the parts. To 
put it in another way, the proper answer to the question why a certain spatial and temporal organisation of parts constitutes a whole is that because the parts together do the same thing what the whole itself does. That is, the overall behaviour of the parts is identical with the behaviour of the whole.

Let us unpack this in detail.

\subsection{Identification of causal roles}

Consider what the relation between the behaviour of the whole and the behaviour of the organised structure of its parts is. This question focuses on the causal role played (the activity performed) by the mechanisms as a whole and the causal role played by the spatial and temporal organisation of the parts.

Remember how mechanistic explanations proceed. First, a certain phenomenon is grasped via the task performed by a system, then the system gets decomposed via the identification of its parts, their activity and organisation. The very point of the mechanistic approach is to explain how a system performs certain tasks by understanding how its parts organised in the right way perform the very same task. Had the organised structure of the lower level entities performed an activity different from what the higher level entity performs, the account of what happens at the lower level would not have been able to explain the higher level phenomenon in question, and the organised activity of the lower level entities would not have constituted the higher level whole. In this sense, mechanistic explanations identify the activity of the spatial and temporal organisation of the parts with the activity of the higher level whole.

That is, the very way mechanistic explanations proceed requires the activity of the organisation of the parts at the lower level to be the same as the activity of the whole at the higher level.

Consider Figure 2, which is a slightly altered version of Figure 1. Figure 2 indicates certain activities of the entities playing part in a mechanistic explanation. Let's say that the entity at the higher level is connected to its context (other entities at its level) by causal relations $C_{i}, C_{j}, C_{k}{ }^{4}$, whereas the lower level entities in question are connected to their context by causal relations $\mathrm{C}_{1}$, $C_{m}, C_{n}$. These causal relations characterise the causal role played by the higher level entity and the organised structure of the lower level entities respectively.

\footnotetext{
${ }^{4}$ One who is subscribed to Shoemaker's analysis $(2003,2007)$ might want to say that $C_{i}$ and $C_{j}$ represent backward looking whereas $\mathrm{C}_{\mathrm{k}}$ represents forward looking causal powers of $\mathrm{X}_{3}$.
} 
The very fact that it is possible to explain the task performed by the higher level entity in terms of the organised activity of the lower level entities entails that the causal roles played at the higher level $\left(C_{i}, C_{j}, C_{k}\right)$ and at the lower level $\left(C_{1}, C_{m}, C_{n}\right)$ are the same. That is, for a mechanistic explanation to get off the ground the causal profile characterised by $C_{i}, C_{j}, C_{k}$ must be identical with the causal profile characterised by $\mathrm{C}_{1}, \mathrm{C}_{\mathrm{m}}, \mathrm{C}_{\mathrm{n}}$. It is thus an internal consequence of the very way mechanistic explanations work that causal connections utilised to characterise the activities at different levels must be identical with each other.

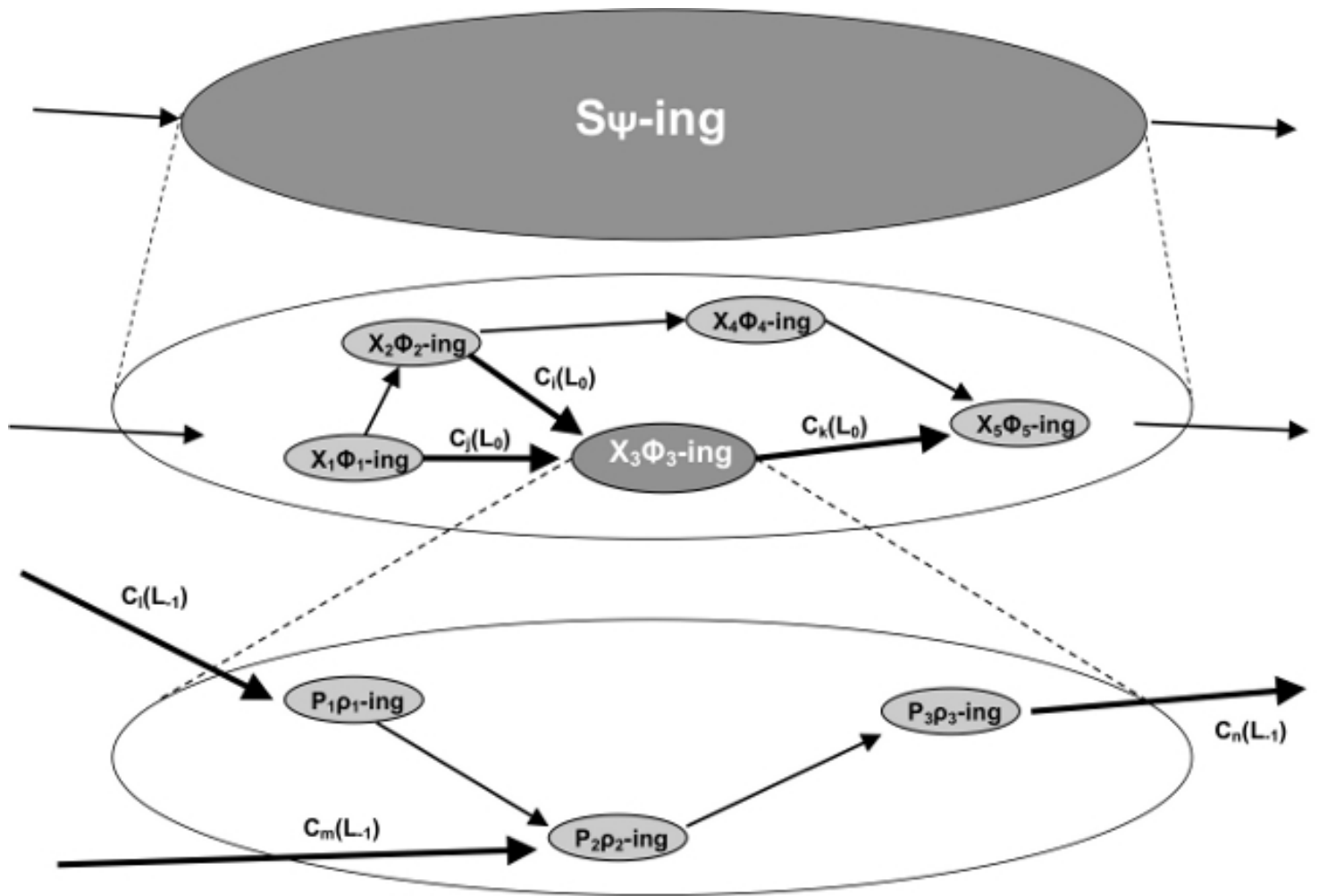

Fig. 2 An inherent commitment of mechanistic explanations.

Causal connections $\mathrm{C}_{\mathrm{i}}, \mathrm{C}_{\mathrm{j}}, \mathrm{C}_{\mathrm{k}}$ of entity $\mathrm{X}_{3}$ belong to the higher level $\mathrm{L}_{0}$, whereas causal connections $C_{1}, C_{m}, C_{n}$ of the organised structure of entities $P_{1}, P_{2}, P_{3}$ belong to the lower level $\mathrm{L}_{-1}$. The mechanistic approach must be committed to the claim that the higher level causal role characterised by $C_{i}, C_{j}, C_{k}$ is identical with the lower level causal role characterised by $\mathrm{C}_{1}, \mathrm{C}_{\mathrm{m}}, \mathrm{C}_{\mathrm{n}}$.

Note that the organisation of the lower level parts would not constitute the higher level whole if their activities weren't identical. That is, the constitution claim the mechanistic approach makes amounts to an identity claim: the causal role played by the organisation of the parts is the 
very same causal role what is played by the whole. The conclusion what follows, then, is this: by claiming that the whole is constituted by the organised activity of its parts the mechanistic approach inherently commits itself to the claim that whatever the whole does is something what is done by the organisation of its parts.

\subsection{Different vocabularies, bridge laws and identity statements}

There is, however, something that seems to be in tension with the conclusion of the last section: literally, entities at higher levels do different things than entities at lower levels. Consider again our example of the descending levels of mechanisms responsible for the task performed by the eye. E.g. at the level of the lens and the retina entities 'focus light rays' and 'send neuronal signals', whereas at the lower level of opsin and transducin there are activities like 'closing ion channels' and 'hyperpolarising'. What the claim that at different levels entities do different things emphasises is the simple fact that e.g. whereas at the higher level there are no entities hyperpolarising, at the lower level there is nothing focusing light rays.

Notice how different vocabularies are utilised in order to describe entities and activities at different levels. As one descends from level to level one needs to change the vocabulary of psychology to the vocabulary of anatomy, then to the vocabulary of neuroscience then to that of molecular biology, and so on. In other words, it is hard to see how a task performed at a higher level could possibly be the same as a task performed at a lower level because different vocabularies are used at different levels to describe entities and activities.

However, it is possible to reconcile the observation that entities at different levels do different things with the constraint that the mechanistic approach inherently commits itself to the claim that the behaviour of the whole is identical with the overall behaviour of the organisation of its parts. What the mechanistic approach needs here are bridge principles. Even if the mechanistic approach subscribes to the functional model of explanation (cf. Craver 2001), which explains a phenomenon via what causal roles it fills, as opposed to the D-N model (explaining a phenomenon via deducing it from general laws and antecedent conditions), bridge principles, identifying different terms of different vocabularies, are still necessary for dealing with specific inter-level relations. (cf. Fazekas 2009)

The need for bridge principles is something Bechtel himself touches upon. He says: 
"Herein lies the explanation for the need for bridge principles in the theory-reduction account - different vocabulary is needed to describe what the parts of a mechanism do than is required to describe what the mechanism as a whole does. The appropriate bridge in this case, however, is not a set of translation rules, but an account of how the operations of the parts of the mechanism are organized so as to yield the behavior of the whole mechanism" (Bechtel \& Hamilton 2007, p. 25, emphases added)

That is, though Bechtel acknowledges that different vocabularies describe activities at different levels, and he also acknowledges that different vocabularies are dealt with by employing bridge principles in the theory-reduction (D-N) account, he still thinks that bridge principles are unnecessary within the mechanistic framework - all one needs is an account of the organisation of the parts' activities.

However, this is quite not right. It is not enough simply to describe the organisation of the constituents. An account of the organisation of the parts' activities is still formulated in the vocabulary of the lower level (e.g. it talks about the spatial and temporal organisation of the transducin activating PDE, the photoreceptor membrane hyperpolarizing, etc.), whereas the behaviours at the higher level are described in another vocabulary used at that particular level (describing how the lens focuses light, the retina converts light into neuronal signal etc.).

Bridge principles need to be evoked here to connect the different vocabularies. These bridge principles express co-reference of different causal terms: they state e.g. that the term 'the eye transducing light causes the optic nerve to signal' refers to the very same causal role than the term 'the photoreceptor hyperpolarises'. They express that the causal roles evoked by descriptions at different levels are the same.

The moral, then, is this. Mechanistic explanations need to incorporate proper identity statements connecting causal roles (activities) at different levels. ${ }^{5}$

\footnotetext{
5 The fact that mechanistic explanations do use identity statements can nicely be pinpointed in some of the texts published by the proponents of the account. For the sake of the example consider the following two quotes.

"In this sketch of events involved in remembering a lecture, I twice stepped down levels by appealing to an identity between the effect on a system and a change in constituents of the system. At the lower level the causal story was an ordinary causal one. Then I stepped up a level by appealing to an identity between the new operations within the mechanism and the way it behaved as a whole. At the level of the whole the story was again an ordinary causal one." (Bechtel 2008, pp. 154-155, emphases added)

"And insofar as that non-functioning constitutes the general's death, we explain her death. Notice that when we reach the state of the mechanism that constitutes the state of death, we do not say, with Betty Crocker, that it causes death. It just is death." (Craver \& Bechtel 2007, p. 557, emphasis added)
} 


\section{Identity Statements and Autonomy}

Our analysis so far points out a commitment of mechanistic explanations: that the appropriate causal processes at higher and lower levels must be identical with each other. This is a general characteristic of all versions of the mechanistic approach. Now we turn our attention to the consequences of this commitment. This section investigates how the commitment in question affects William Bechtel's program of balancing between reduction and autonomy.

As we have seen, Bechtel provides two arguments for the autonomy of higher levels. On the one hand, he claims that organisational information regarding the parts goes beyond the account of the parts and their operations. On the other hand, he claims that contextual information, i.e. information about the interactions the whole enters is not part of the reductive account characterising the components and their operations (cf. Bechtel 2007, pp. 182-183). In the following two sections we shall present why a commitment to identity statements cause problems for both arguments.

Before being immersed into the details, note that for Bechtel these two arguments provide the only support for maintaining the autonomy of higher levels. The most common source of support, the multiple realisability argument, is not an option for him-Bechtel thinks that the multiple realisability claim is mistaken, and argues in length against it (cf. Bechtel \& Mundale 1999; see also Bechtel 2008 pp. 135-142). ${ }^{6}$ That is, he needs alternative criteria to support his autonomy claim. The organisational and contextual information based arguments play this role.

\subsection{Organisation}

We have objected Bechtel's claim that instead of bridge principles connecting the behaviour of a higher level whole to the overall behaviour of its lower level parts all that one needs is an account of how the activities of the parts are organised (cf. Section 3.2). Note that our objection relies on the assumption that (at least there are cases where) the organisation of the activities of the parts is captured in terms of a lower level vocabulary, whereas the behaviour of the whole is captured in a higher level vocabulary.

This however is not what Bechtel thinks about organisational information. Recall what he has to say about the autonomy of higher levels (cf. Section 2.1). Bechtel explicitly argues that in-

\footnotetext{
${ }^{6}$ Note that given Bechtel's commitment against multiple realisability, identity of causal roles — since the mechanistic framework is subscribed to the functional model of reduction (cf. Craver 2001; Bechtel 2008) -implies the identity of the entities at different levels filling the same causal roles.
} 
formation about how the parts are spatially and temporally organised-i.e. the very organisational information in question - goes beyond the account of the parts and their operations.

We think that Bechtel is wrong. To be more precise, we think that either Bechtel is wrong or he defends a trivial claim. The ambiguity derives from how one understands the term "reductive account characterizing the parts and operations within the mechanism" (Bechtel 2007, p. 183), for this is the term which refers to that lower level characterisation which-according to Bechtel-is unable to capture organisational information.

One way to understand this term is interpreting it as a characterisation of individual lower level entities and their activities. According to this understanding, then, Bechtel's claim reads as follows. Organisational information of lower level entities and their activities is not part of the characterisation of these entities as taken individually. This is the trivial claim. It is trivial because, whereas the accused characterisation is restricted solely to individual lower level entities and the causal roles they fill, the shortcoming it is accused with is the inability to account for spatial and temporal relations between these individual entities. Consider, again, our example. No matter how detailed description is given about the intrinsic properties of the iris, the pupil, the lens, the ciliary muscles and the retina, and the tasks they are able to perform individually, this description necessarily lacks all the information about how these entities are connected, what spatial and temporal structure they fit into, and what dynamical couplings take place between their activities.

However, it does not follow that it is impossible to capture this information purely at the level of these entities. For there is another way to understand the term above- as a full characterisation of the lower level. According to this understanding, the reductive account characterising the lower level involves everything that can be described in terms of the lower level vocabulary. We see no reason why spatial, temporal and relational facts about the iris, the pupil etc. could not be captured in terms of the vocabulary of their level. On the contrary: it is exactly this level - as opposed to the higher level, as Bechtel claims - which is able to account for these spatial, temporal and relational facts, since it is the vocabulary of this level which readily possess the very terms 'iris', 'pupil', etc., and thus is most naturally apt for capturing such facts. In fact, it seems that it is impossible to capture the organisation of lower level entities at the higher level. Spatial, temporal and relational facts of lower level entities can only be discovered and described by employing the methodology and vocabulary of the lower level. That is, if Bechtel's claim is that information about 
the organisation of lower level entities cannot be captured at the lower level then it is strictly false (or questionable at best, and in need for more support).

The moral is that Bechtel's organisation-based argument for the autonomy of the higher levels does not go through. In fact, it attacks a straw man: it is true (though trivially) if one restricts the characterisation of the lower level to the characterisation of individual entities and their behaviour. However, we see no reason why the targets of this argument, reductive accounts, should restrict themselves to this 'light' way of characterising the lower level. In fact, John Bickle (2003), who is at the extreme reductionist end of the reduction-autonomy axis (cf. Section 2.2) is quite explicit that lower level accounts are full in this sense: they characterise not only the entities taken individually but their relations as well. That is, full-blown lower level accounts incorporating everything describable in terms of the lower level vocabulary are left intact by Bechtel's argument.

Bechtel might object here by further claiming that although providing a full description at the lower level is possible, it still fails to capture the relevant aspects of the organisation, since the whole functions properly only in the appropriate context, and this contextual information is not provided even by a full-blown lower level description of the organisation. In this case, then, his first claim for the autonomy of higher levels collapses into his second claim.

\subsection{Context}

The second claim Bechtel makes argues for the autonomy of higher levels by stressing that contextual information about how a higher level whole is related to other higher level entities in its environment is inaccessible at the lower level (at the level of the parts).

This claim reminds us to the previous one we have just discussed in the following sense. In the preceding section we have pointed out that Bechtel is attacking a straw man by restricting the characterisation of the lower level to the characterisation of individual entities and their behaviour. He makes the same mistake again. Only in this case it is not the relations between the parts of the whole what he illegitimately excludes from the characterisation of the lower level, but other entities of the lower level and their activities. These lower level entities in question are not parts of the original higher level whole but parts of other higher level entities that are connected to the original whole.

In other words, the mistake Bechtel makes here is misidentifying the supervenience base. In the case of organisational information it seems as though Bechtel asked the reductionists to explain 
organisational facts solely on the grounds of facts about lower level entities and their activities as taken individually, but not their spatial and temporal relations. In the case of contextual information it seems as though he asked the reductionists to explain contextual facts about the higher level solely on the grounds of facts about the lower level which exclude the so-called supervenience base of the context itself. Contextual information about the connections of the higher level whole supervenes not just on the parts of the whole, but on other entities of the lower level as well-in fact on all those entities of the lower level which themselves are parts of other higher level entities being connected to the very whole in question.

Consider Figure 3. It is an amended version of Figure 1. It shows that just as the activity of a given higher level entity (in this case the $\Phi_{3}$-ing of $\mathrm{X}_{3}$ ) can be accounted for in terms of the organisation of the activities of lower level entities ( $P_{1} \rho_{1}$-ing, $P_{2} \rho_{2}$-ing, and $P_{3} \rho_{3}$-ing), it is also possible to account for other higher level entities (e.g. $\mathrm{X}_{2}$ and $\mathrm{X}_{5}$ ) - in the very same way-in terms of other entities and activities at the lower level. Take, for example, our model case. In addition to accounting for how the retina works in molecular terms, it is also possible to account for how the iris can vary the size of the pupil, or how ciliary muscles adjust the shape of the lens in terms of the very same vocabulary of molecular biology.

Remember, the mechanistic approach is committed to the identification of higher and lower level causal connections. At the higher level, there are causal relations connecting the higher level entities to each other. Each higher level entity can be accounted for in terms of the organised structure of lower level entities. The inherent commitment of the mechanistic framework implies that the causal relations connecting a higher level entity to other entities at that level are identical with causal connections connecting certain lower level entities constituting the higher level entity in question with other lower level entities constituting those higher level entities which the original higher level entity is connected with. The way lower level entities constituting one higher level entity are connected to other lower level entities constituting other higher level entities captures the higher level context in terms of lower level facts. That is, along the lines of the identities of causal roles, it is possible to capture higher level contextual information at the lower level. 


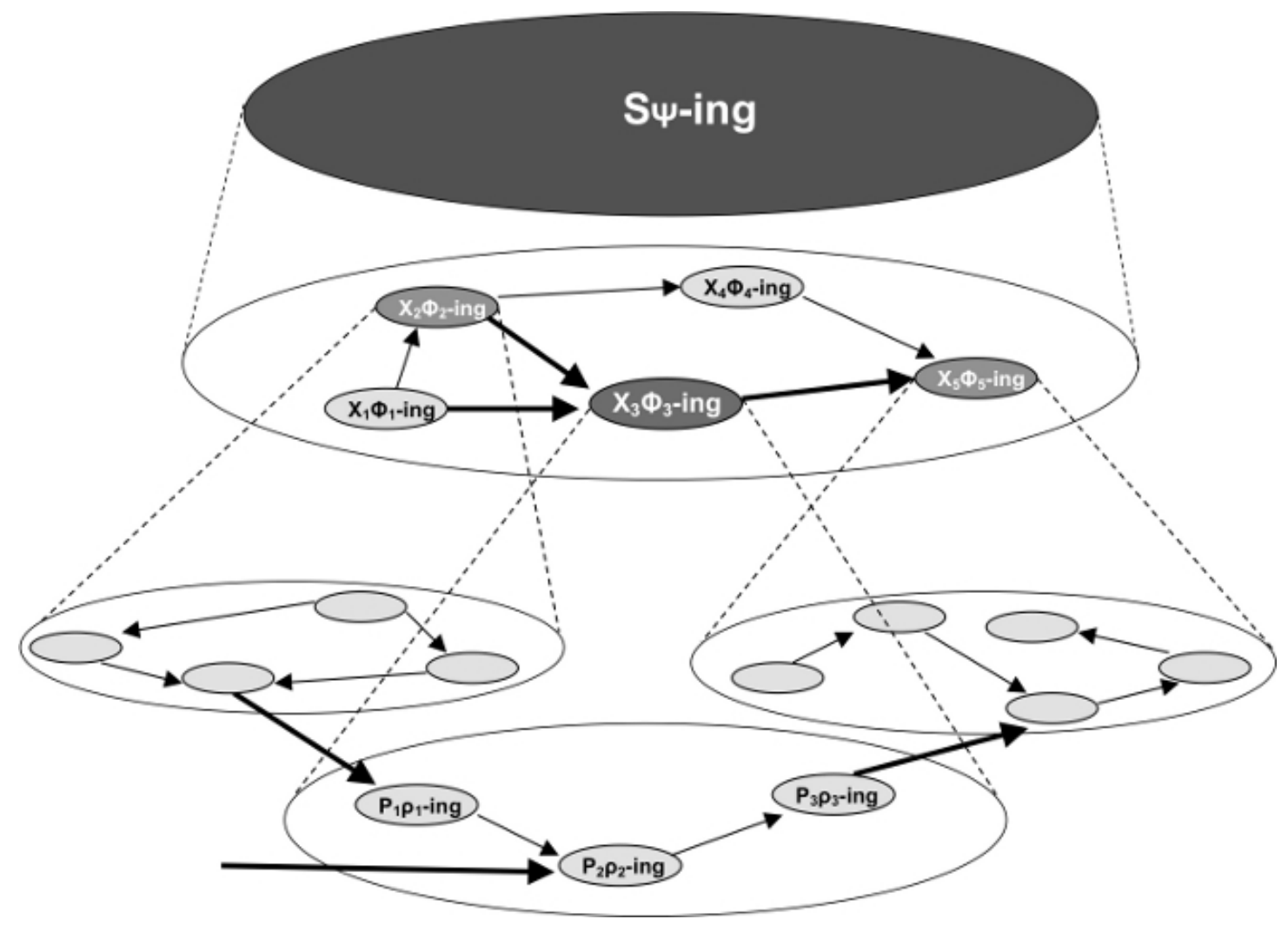

Fig. 3 Context on lower levels.

Contextual information can be grasped on the lower level if lower level constituents of higher level entities providing the context are taken into consideration.

One might object here claiming that our analysis above is in direct tension with some of Bechtel's and Craver's general remarks on the characteristics of mechanistic explanations. They both emphasise that the levels of mechanisms are defined locally. Bechtel, for example, claims that "because of the lack of a compositional relation between the subparts of two different working parts of the mechanism, the question of whether these are at the same level is not well defined" (Bechtel 2008, p. 147). According to Craver, $X$ and $S$ are at the same level of mechanisms only if $X$ and $S$ are components in the same mechanism, and neither the activity of $\mathrm{X}$ is a component of the activity of S, nor vice versa. Defining levels of mechanisms locally entails that when two entities of the same level are further decomposed into parts then there is no warranty that the parts constituting one of the higher level entities are at the same level as those which constitute the other higher level entity (cf. Craver 2007, pp. 192-194). Since this is exactly what our line of thought above assumes, it seems that our argument for capturing the higher level context at the lower level is bound to fail. 
However, we think that there are good reasons for believing that locally defined levels can be extended in a way that it becomes intelligible to claim that constituent parts of different higher level entities are indeed at the same level.

First, consider what Craver and Bechtel (2007) say about causation. They argue that causation is an intra-level relation (cf. Section 2.3). That is, if there were causal connections between entities constituting different higher level wholes, then those two entities — and in turn all the constituent entities of both lower level mechanism responsible for the higher level wholes respectively-would be at the same level even if originally they were defined only locally.

Second, the very same methodology which is utilised when investigating what lower level entities play a part in the mechanism responsible for the higher level phenomenon can just as well be utilised in order to test if a lower level entity constituting a higher level entity is connected to another lower level entity constituting another higher level entity (which is causally connected to the first higher level entity). Note that such causal connections between lower level entities constituting distinct higher level wholes are well known: consider, for example, how molecular processes in the retina (partly constituting the eye) affect molecular processes in the optic nerve (partly constituting the nervous system).

Third, consider Craver's own definition according to which two entities are at the same level if they are components of the same mechanism. Compare this with Figure 3. True, the organised structures of the lowest level entities (lowest, as depicted on Figure 3, not in an absolute sense) are defined locally via decomposing different middle level entities. Nonetheless, pairs of the sets of lowest level entities (forming organised activities at the lowest level and thus being responsible for the activities of certain middle level entities) are components of the same mechanism responsible for the middle level phenomenon of a certain middle level entity affecting another middle level entity (say, the $\Phi_{3}$-ing of $X_{3}$ affecting the $\Phi_{5}$-ing of $X_{5}$ on Figure 3 ). For example, molecular processes in the retina and molecular processes in the optic nerve together are components of the same mechanism responsible for the higher level phenomenon that the eye transducing light causes neuronal activity in the nervous system.

In fact, the conclusion that there is an extended lower level containing distinct constituents of causally connected higher level entities follows from the fact that the mechanistic approach is committed to the identity of causal roles. For the only way a lower level causal connection can really be identical with a higher level one is if the lower level entity connected to the constituent of 
one of the higher level entities being causally connected is indeed a constituent of the other higher level entity. Had it been a lower level entity whose activity is entirely indifferent for the higher level entities in question, the lower level connection would not have been identical with the original higher level connection. This, in turn, guarantees that the lower entities causally connected at the lower level are constituents of the higher level entities respectively, and the fact that there is a causal connection between them guarantees that they are at the same level. The lower level extended in this way, then, would be able to capture the contextual information in question.

That is, contrary to what Bechtel claims, it is possible to account for contextual information about higher levels in terms of the entities and activities of lower levels. What one needs in order to be able to do so is recognising that all lower level entities and activities corresponding to higher level contexts must be included into the lower level account. ${ }^{7}$

Surely, one cannot tell what higher level processes are taking place solely on the ground of what one knows about the lower level. However, it is due solely to the difference in the vocabularies describing the two levels. Once the required identity statements are in place one is able to infer to higher level processes from lower level knowledge. Bechtel is right that one needs the higher level as well- but only for formulating identity statements. And this is true of the more extreme reductionist accounts denying the autonomy of higher levels (like classical theory reduction, Kimian functional reduction, or Bickle's account—cf. Fazekas 2009) as well.

That is, William Bechtel's approach cannot maintain the autonomy of higher levels any better than full-blown reductive accounts do. If one understands autonomy as claiming that in order to explain a certain phenomenon one needs the higher level as well, then, though true, it is a quite weak sense of autonomy. (Weak, since it is also true of even some quite radical versions of reductionism.) If, contrary to this, one understands autonomy as claiming that at higher levels it is possible to account for facts which cannot be captured at lower levels, then, though this is a more attractive and informative version of autonomy, it is simply not true that Bechtel-style mechanistic explanations support maintaining the autonomy of higher levels in this sense.

\footnotetext{
${ }^{7}$ A somewhat similar point has been suggested by Megan Delehanty (2005) under the term of 'mechanism extension'.
} 


\section{Identity Statements and Downward Causation}

Finally, before concluding the paper, let us briefly come back to downward causation and the account Craver and Bechtel (2007) provides. The previous section investigated the consequences of embracing identities of causal roles to Bechtel's program. This section extends the investigation to Craver and Bechtel's aim of making downward causation intelligible.

The basic claim Craver and Bechtel make is that downward causation (and inter-level causation in general) is intelligible. There is no mystery concerning how higher level entities affect lower level entities causally — as far as one appreciates the mechanistic framework, i.e. as far as one bears in mind that there is no direct causal connection between higher level entities and lower level ones. What there is, instead, is the constitution relation 'mediating' the effects of the higher level down to the lower level.

Claiming that higher level causes mediated down to lower levels make downward causation intelligible seems to suggest that there is higher level causation 'over and above' (i.e. different from) the causation at the lower level. For what else could be 'mediated' down to the lower level if all causal connections present at the higher level already existed at the lower level?

However, this picture is confusing given our analysis of the constitution relation and the commitment of the mechanistic approach to identifying causal connections at higher levels with causal connections at lower levels. The mechanistic approach inherently identifies the behaviour of the higher level whole with the behaviour of the organisation of its parts. If so, then all the causal influence higher level entities are able to exert are already exerted by lower level entities.

Therefore, downward causation can be made intelligible in only one (quite restricted) sense. A cause affecting a higher level whole affects its parts because the cause in operation at the higher level is identical with a cause operating at the lower level affecting the parts in question. This, however, is typically not how downward causation is generally understood. The fundamental tenet proponents of downward causation defend is that there are novel causal powers at higher levels. Novel either in the stronger ontological sense that they are not even metaphysically determined by the causal powers of the lower level, or in the weaker epistemological sense that they cannot be accounted for in terms of the causal powers at the lower levels (Alexander 1920; Broad 1925; Kim 1999, 2000; McLaughlin 1992; Morgan 1923; Stephan 2002; Yates 2009). Neither of these requirements are fulfilled, though, within the mechanistic framework. The mechanistic ap- 
proach - contra the ontological understanding of downward causation-identifies higher level causal connections with lower level ones, and - contra the epistemological understanding-claims that the behaviour of higher level wholes can be accounted for in terms of the behaviour of their lower level parts.

That is, the mechanistic approach entails that downward causation, as it is usually understood, does not exist. What interpreting downward causation within the mechanistic framework can achieve at best is explaining why people often think that those phenomena they come across are cases of downward causation. Strictly speaking, the mechanistic framework is inapt to explain downward causation - it explains it away.

\section{Conclusion}

The primary objective of this paper was to track the commitments of mechanistic explanations regarding the relation between causal connections at different level. We have shown that mechanistic explanations embrace identity statements identifying higher level causal connections with lower level ones. Next, we have investigated the consequences of this commitment to William Bechtel's program of balancing between the reducibility and autonomy of higher levels. In the course of this endeavour, we have pointed out that locally defined levels can be extended to contain entities which are not part of the locally defined mechanism but constituents of higher level entities causally connected to the original higher level entity. Relying on this result, we have concluded that Bechtel, once giving up on multiple realisability and being committed to 'mechanistic reduction' looses all resources to maintain the autonomy of higher levels. Finally, we have examined a recent attempt to interpret downward causation from the point of view of the mechanistic approach. We have made it explicit that the mechanistic framework entails the non-existence of downward causation - though it is able to show what it is that usually gets misidentified as downward causation.

\section{Acknowledgments}

Thanks to George Kampis, Jonas Christensen, Jesper Kallestrup, Kim Sterelny and an anonymous referee for their valuable comments. One of the authors (PF) is grateful to the Eötvös Scholarship 
from the Hungarian State and the University of Edinburgh College of Humanities and Social Sciences Scholarship for their generous support.

\section{Bibliography}

Alexander, S. (1920) Space, Time, and Deity. London: Macmillan.

Baker, L. R. (1997) Why Constitution is not Identity. J Philos 94:599-621.

Bear, M. F., Connors, B. W. \& Paradiso, M. A. (2001) Neuroscience: Exploring the Brain. Baltimore: Lippincott Williams \& Wilkins.

Bechtel, W. (2006) Discovering cell mechanisms: the creation of modern cell biology. New York: Cambridge University Press.

Bechtel, W. (2007) Reducing psychology while maintaining its autonomy via mechanistic explanation. In: M. Schouten \& H. Looren de Jong (Eds) The Matter of the Mind: Philosophical Essays on Psychology, Neuroscience and Reduction. Oxford: Basil Blackwell, pp. 172-198

Bechtel, W. (2008) Mental mechanisms: philosophical perspectives on cognitive neuroscience. New York: Lawrence Erlbaum Associates.

Bechtel, W. \& Hamilton, A. (2007) Reduction, integration, and the unity of the sciences. In: T. Kuipers (Ed) Philosophy of Science: Focal Issues (Vol. 1 of the Handbook of the Philosophy of Science). New York: Elsevier

Bechtel, W. \& Mundale, J. (1999) Multiple realizability revisited: Linking cognitive and neural states. Philos Sci 66(2):175-207.

Bedau, M. (2002) Downward Causation and the Autonomy of Weak Emergence. Princ 6(1):5-50.

Bickle, J. (2003) Philosophy and neuroscience: A ruthlessly reductive account. Drodrecht: Kluwer Academic Publishing.

Bickle, J. (2006) Reducing mind to molecular pathways: explicating the reductionism implicit in current cellular and molecular neuroscience. Synth 151:411-434.

Broad, C. D. (1925) The Mind and Its Place in Nature. London: Routledge and Kegan Paul.

Craver, C. F. (2001) Role Functions, Mechanisms, and Hierarchy. Philos Sci 68(1):53-74.

Craver, C. F. (2007) Explaining the brain: mechanisms and the mosaic unity of neuroscience. Oxford: Clarendon Press

Craver, C. F. \& Bechtel, W. (2007) Top-down Causation Without Top-down Causes. Biol Philos 22(4):547-563.

Delehanty M. (2005) Emergent Properties and the Context Objection to Reduction. Biol Philos 20(4):715-734.

Fazekas, P. (2009) Reconsidering the role of bridge laws in inter-theoretic reductions. Erkenn 71:303-322.

Glennan, S. (1996) Mechanisms and the nature of causation. Erkenn 44: 49-71

Glennan, S. (2009) Mechanisms, Causes, and the Layered Model of the World. Philos and Phenomenol Res, forthcoming, 1-29.

Johnston, M. (1992) Constitution is not Identity. Mind 101:89-105

Kandel, E. R., Schwartz, J. H. \& Jessell, T. M. (2000) Principles of Neural Science. New York: McGraw-Hill

Kim, J. (1999) Making Sense of Emergence. Philos Stud 95:3-36 
Kim, J. (2000) Making sense of downward causation. In: P. B. Andersen, C. Emmeche, N. O. Finnemann \& P. V. Christiansen (Eds) Downward causation, Aarhus University Press, pp. 305321.

Machamer, P., Darden, L. \& Craver, C. (2000) Thinking about mechanisms. Philos Sci 67:1-25.

McLaughlin, B. P. (1992) The Rise and Fall of British Emergentism. In: A. Beckermann, H. Flohr \& J. Kim (Eds) Emergence or Reduction? Essays on the Prospects of Non-reductive Physicalism, Berlin: De Gruyter, pp 49-93

Menzies, P. (2003). The Causal Efficacy of Mental States. In: S. Walter and H.D. Heckmann (Eds) Physicalism and Mental Causation. Imprint Academic, pp. 195-224.

Menzies, P. (2010) The Exclusion Problem, the Determination Relation, and Contrastive Causation. In: J. Hohwy and J. Kallestrup (Eds) Being Reduced: New Essays on Reductive Explanation and Special Science Causation. Oxford University Press, pp 196-217.

Morgan, L. (1923) Emergent Evolution. London: Williams \& Norgate

Paul, L. A. (2007) Constitutive Overdetermination. In: J. K. Campbell (Ed) Topics in Contemporary Philosophy vol. 4: Causation and Explanation, Cambridge: MIT Press

Shoemaker, S. (2003) Realization, Micro-realization, and Coincidence. Philos Phenomenol Res 67:1-23

Shoemaker, S. (2007) Physical Realization. Oxford: Oxford University Press

Stephan, A. (2002) Emergentism, Irreducibility, and Downward Causation. Grazer Philos Stud 65:77-93

Yates, D. (2009) Emergence, downwards causation and the completeness of physics. Philos Q 59(234):110-131 